\title{
Insuffisance rénale aiguë et syndrome hépatorénal chez le patient cirrhotique : actualités diagnostiques et thérapeutiques
}

\author{
Acute Kidney Injury and Hepatorenal Syndrome in patients with cirrhosis: \\ diagnostic and therapeutical novelties
}

\author{
M. Khaldi · E. Lemaitre - A. Louvet · F. Artru \\ Reçu le 30 juillet 2018; accepté le 22 novembre 2018 \\ (C) SRLF et Lavoisier SAS 2018
}

Résumé La survenue d'une insuffisance rénale aiguë ou AKI (acute kidney injury) chez un patient cirrhotique est un événement de mauvais pronostic. Parmi les AKI, une entité spécifique au patient cirrhotique décompensé est le syndrome hépatorénal (SHR) dont la définition ainsi que la stratégie thérapeutique ont été réactualisées récemment. La prise en charge de l'AKI hors SHR n'est pas spécifique au patient cirrhotique. La prise en charge du SHR repose sur l'association d'un traitement vasoconstricteur intraveineux et d'un remplissage vasculaire par sérum d'albumine concentrée. Cette association thérapeutique permet d'améliorer le pronostic des patients répondeurs. En contexte d'AKI chez le patient cirrhotique, l'épuration extrarénale (EER) peut être envisagée en cas de non-réponse au traitement médical. La décision de débuter une prise en charge invasive avec EER dépend principalement de la présence d'un projet de transplantation hépatique (TH). En l'absence d'un tel projet, cette décision devrait être prise après évaluation du pronostic à court terme du patient dépendant du nombre de défaillance d'organes et d'autres variables telles que l'âge ou les comorbidités. L'objectif de cette mise au point est de discuter des récentes modifications de la définition de l'AKI et en particulier du SHR chez les patients cirrhotiques, de détailler la prise en charge spécifique du SHR et d'évoquer les processus décisionnels menant ou non à l'instauration d'une EER chez les patients non répondeurs au traitement médical en milieu réanimatoire.

Mots clés Insuffisance rénale aiguë · Syndrome hépatorénal · Syndrome acute on chronic liver failure Cirrhose $\cdot$ Transplantation hépatique

\footnotetext{
M. Khaldi · E. Lemaitre · A. Louvet · F. Artru $(\bowtie)$ Unité Inserm U995, service des maladies de l'appareil digestif, unité des soins intensifs hépatogastroentérologique, hôpital Claude-Huriez, CHU de Lille, F-59037 Lille, France e-mail : florent.artru@chru-lille.fr
}

\begin{abstract}
Occurrence of acute kidney injury (AKI) in cirrhotic patients is associated with poor outcome. Among AKI, hepatorenal syndrome (HRS) is a particular entity which is observed only in severe cirrhotic patients disclosing high level of portal hypertension and hepatocellular insufficiency. Definitions for AKI, HRS-AKI and specific medical management have been recently updated. HRS corresponds to an AKI with a functional profile associated with a high shortterm mortality rate in the absence of response to medical treatment. This latter associates albumin perfusion and vasoactive treatment such as terlipressin. This treatment improves short-term prognosis of responders to medical therapy. In case of treatment failure, renal replacement therapy (RRT) can be proposed. Decision-making process leading either to propose RRT or not depends mainly on eligibility to do liver transplantation. In absence of such eligibility assessed by local expertise, decision to start RRT should be based on the prognosis assessed by the number of organ failures and other variables such as age and comorbidities. The aims of this article are to discuss the new definition of AKI and AKI-HRS, treatment strategy particularly in the HRS setting and in patients who are not responding to medical therapy leading to take decision or not to start RRT.
\end{abstract}

Keywords Acute kidney injury · Hepatorenal syndrome · Acute-on-chronic liver failure syndrome $\cdot$ Cirrhosis · Liver transplantation

\section{Introduction}

La cirrhose est une pathologie fréquente et grave touchant environ 700000 malades en France et responsable de 15000 décès par an [1]. On estime que 2,7 à $5,1 \%$ de la population totale des patients hospitalisés en réanimation présentent une cirrhose. La prévalence de cette pathologie varie de manière importante selon les centres et leur expertise 
[2-4]. Il existe une augmentation du nombre d'admissions de ces patients en réanimation, représentant $1,6 \%$ de la population admise en réanimation en 1998 à 3,1\% en 2012 au Royaume-Uni [3]. Le pronostic de ces patients s'est rapidement amélioré ces dernières années. À titre d'exemple, il a été rapporté un gain de survie d'environ 20 points à 28 jours entre 2000 et 2010 (40\% en 2000 à $63 \%$ en 2010) [5]. Cela est lié en particulier à une amélioration et à une standardisation des prises en charge hépatologique [6-9] et réanimatoire [10,11]. La survenue d'une insuffisance rénale aiguë (IRA) dans un contexte de cirrhose est associée à un mauvais pronostic à court terme. L'IRA touche environ $20 \%$ des patients cirrhotiques décompensés admis en hospitalisation [12]. La créatininémie, reflet du débit de filtration glomérulaire, est d'ailleurs une des trois variables (avec l'INR et la bilirubinémie) permettant de calculer le score MELD (Model for End-stage Liver Disease) validé internationalement comme score prédictif de mortalité à trois mois [13-15] et utilisé dans le score d'allocation des greffons. Le syndrome hépatorénal (SHR) est une forme spécifique d'IRA du patient présentant une cirrhose décompensée dont les critères diagnostiques et la prise en charge thérapeutique ont été modifiés récemment [16-18] et dont l'incidence annuelle est élevée ( $8 \%$ ) chez le patient cirrhotique décompensé [19]. Sur le plan physiopathologique, le SHR correspond à une insuffisance rénale au profil fonctionnel résultant de plusieurs phénomènes liés à l'hypertension portale et à l'insuffisance hépatocellulaire présents chez les patients cirrhotiques décompensés (vasodilatation artérielle splanchnique, translocation bactérienne, hypovolémie efficace, troubles microcirculatoires) [20]. La survenue d'une IRA peut être associée à une défaillance hépatique ou à d'autres défaillances d'organes extrahépatiques s'inscrivant dans le syndrome d'acute-on-chronic liver failure (ou ACLF) dont le pronostic dépend du nombre de défaillances d'organes [21]. De fait, la prise en charge thérapeutique de l'insuffisance rénale devra s'inscrire dans une prise en charge plus globale définie par la présence d'un projet de transplantation hépatique $(\mathrm{TH})$ et le pronostic du patient évalué par le nombre de défaillances d'organes.

Dans cette mise au point, nous détaillerons les nouveaux critères diagnostiques de l'IRA (ou AKI [acute kidney injury]) et du SHR ainsi que leur prise en charge. Nous évoquerons la place de l'épuration extrarénale (EER) en fonction, en particulier, de la perspective d'une TH à court terme et du pronostic du patient évalué par le nombre de défaillances d'organes.

\section{Classification actualisée de l'insuffisance rénale aiguë : AKI (acute kidney injury)}

Les critères diagnostiques de l'insuffisance rénale aiguë (AKI) ont été révisés récemment par le groupe KDIGO (Kidney
Disease Improving Global Outcomes) et validés par l'International Club of Ascitis (ICA) [16]. Elle se définit par une augmentation de la créatininémie de $26,5 \mu \mathrm{mol} / 1$ en 48 heures ou de $50 \%$ en sept jours (la valeur de base de la créatininémie utilisée doit dater de moins de trois mois ; celle de l'admission sera utilisée en cas d'absence de valeur de référence) [18]. Les critères KDIGO incluent également l'oligurie dans la définition et les différents stades AKI, mais elle n'a pas été retenue par l'ICA, car la diurèse est difficilement interprétable chez les patients cirrhotiques. Du fait d'une rétention hydrosodée importante, une oligurie est en effet souvent présente chez ces patients sans altération du débit de filtration glomérulaire [20]. De plus, ces patients sont souvent traités par diurétiques modifiant donc artificiellement la diurèse.

Trois stades AKI ont été définis selon la gravité et la cinétique d'aggravation et sont de pronostics différents (Tableau 1). Au sein du stade 1, les stades 1A (définis par une créatininémie $\leq 133 \mu \mathrm{mol} / \mathrm{l}$ ) et $1 \mathrm{~B}$ (créatininémie $\geq 133 \mu \mathrm{mol} / \mathrm{l}$ ) ont été distingués du fait de pronostics différents à court terme. Les patients présentant une AKI de stade 1A ont le meilleur pronostic avec une survie supérieure à $80 \%$ à trois mois alors qu'en cas de stade $1 \mathrm{~B}$ celle-ci n'est évaluée qu'entre 55 et $68 \%$ [16,22,23]. Le pronostic le plus sévère est présenté par les patients avec AKI stades 2 et 3 dont la survie à trois mois est respectivement de 42 et $31 \%$ [23].

Les étiologies d'AKI chez le patient cirrhotique sont représentées par l'insuffisance rénale fonctionnelle par hypovolémie (27-50\%), l'AKI liée au sepsis ou à une cause parenchymateuse (14-35\%) et le SHR (15-43\%) [24].

L'étiologie est déterminante dans le pronostic de ces patients. En effet, le pronostic est plus sévère en cas d'AKI survenant dans un contexte de SHR (survie à trois mois de 15 à $37 \%$ ) ou d'AKI liée au sepsis (survie à trois mois de 31 à $58 \%$ ) qu'en cas d'hypovolémie (survie à trois mois de 46 à $65 \%$ ) ou de cause parenchymateuse autre (survie à trois mois de 73 à $76 \%$ ) [23,25].

\section{Syndrome hépatorénal (SHR)}

Le SHR est une maladie fréquente représentant environ un tiers des AKI chez les patients cirrhotiques décompensés hospitalisés pour une complication de la pathologie hépatique $[24,26]$. La physiopathologie du SHR est liée à l'hypertension portale et à l'insuffisance hépatocellulaire du patient cirrhotique et résulte de l'association d'une dysfonction circulatoire et d'une inflammation systémique. L'hypertension portale est responsable d'une translocation bactérienne activant l'immunité innée à l'origine d'un relargage systémique de cytokines pro-inflammatoires, mais également de vasodilatateurs tels que le monoxyde d'azote (NO) $[20,27]$. La translocation bactérienne est aussi responsable 
Tableau 1 Définition, stades et pronostic de l'acute kidney injury [22,23]

\begin{tabular}{|c|c|c|}
\hline Stade AKI & Définition & Survie à 90 jours $(\%)$ \\
\hline Stade 1 & $\begin{array}{l}\text { Augmentation du taux de créatinine } \geq \mathbf{2 6 , 5} \boldsymbol{\mu m o l} / \mathbf{l} \text { en } 48 \text { h ou valeur } \\
\text { de créatinémie }>\mathbf{1 , 5}-\mathbf{2} \text { fois la valeur de créatinine de base } \\
\text { Stade } 1 \mathrm{~A}: \text { créatininémie } \leq 133 \mu \mathrm{mol} / 1 \\
\text { Stade } 1 \mathrm{~B}: \text { créatininémie } \geq 133 \mu \mathrm{mol} / 1\end{array}$ & $\begin{array}{l}84 \\
68\end{array}$ \\
\hline Stade 2 & Valeur de créatininémie à $2-3$ fois la valeur de créatinine de base & 42 \\
\hline Stade 3 & $\begin{array}{l}\text { Valeur de créatininémie } \geq 3 \text { fois la valeur de créatinine de base } \\
\text { ou créatininémie } \geq 364,5 \mu \mathrm{mol} / \mathrm{l} \text { avec augmentation du taux } \geq 26,5 \mu \mathrm{mol} / \mathrm{l} \\
\text { ou nécessité d'une EER }\end{array}$ & 31 \\
\hline
\end{tabular}

d'une dysfonction microcirculatoire et d'une inflammation locale se compliquant d'un stress oxydatif (lié à l'effet des DAMPs ou danger-associated molecular patterns et PAMPs ou pathogen-associated molecular patterns) sur les cellules des tubules rénaux favorisant l'altération de la capacité de filtration glomérulaire [20].

Ces phénomènes favorisent une vasodilatation artérielle splanchnique et une dysfonction cardiocirculatoire (avec cardiomyopathie caractérisée par une dysfonction diastolique, une altération de la fonction systolique en réponse au stress et des anomalies électrophysiologiques) [24] responsables d'une hypovolémie efficace. En réponse, on observe une activation des systèmes vasoconstricteurs tels que le système nerveux sympathique et le système rénine-angiotensine aboutissant à une diminution de la perfusion rénale [20,27]. Chez le patient cirrhotique, il existe par ailleurs une vasoconstriction artérielle intrarénale excessive liée à un défaut de production de vasodilatateurs rénaux participant à la physiopathologie du SHR [20].

L'ensemble de ces mécanismes intriqués (Fig. 1) permettent d'expliquer que le SHR corresponde à une insuffisance rénale d'allure fonctionnelle avec oligurie et natriurèse diminuée. Le diagnostic de SHR reste cependant un diagnostic d'élimination, et plusieurs critères ont été développés et validés par l'ICA afin d'aider au diagnostic [18,20,28]. L'ensemble des critères doivent être présents pour retenir le diagnostic de SHR (Tableau 2).

Deux types de SHR étaient antérieurement décrits selon la rapidité d'installation de l'insuffisance rénale. Le SHR de type 1 correspondait à l'installation rapidement progressive, en moins de 15 jours, d'une IRA (définie par un doublement du taux de créatininémie) et dont le taux devait être supérieur à $220 \mu \mathrm{mol} / 1$. Le SHR de type 2 était défini par une installation plus lente de la dysfonction rénale le plus souvent en contexte d'ascite réfractaire. La définition de ces deux types de SHR est remise en cause actuellement. La modification des définitions a été en partie argumentée par l'absence de prise en charge thérapeutique avant que la créatininémie atteigne $220 \mu \mathrm{mol} / 1$. Plusieurs études ont en effet montré qu'un taux élevé de créatininémie était un facteur de

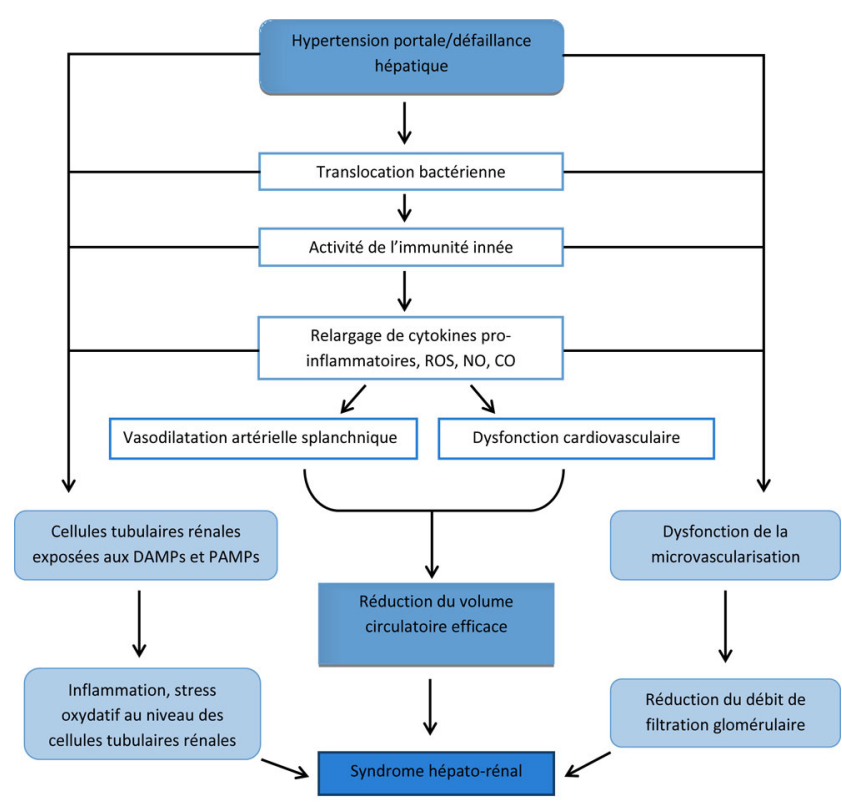

ROS: reactive oxygen species; NO: nitric oxide; CO: carbon monoxide; DAMPs: dangerassociated molecular patterns; PAMPs: pathogen-associated molecular patterns

Fig. 1 Physiopathologie du SHR (adaptée de Piano et al. [20])

Tableau 2 Critères diagnostiques du SHR (adapté de [28]

Critères diagnostiques du syndrome hépatorénal

Diagnostic de cirrhose et présence d'ascite

Présence d'une insuffisance rénale aiguë $\geq$ au stade 2 d'AKI Absence d'utilisation récente de produits néphrotoxiques Absence de réponse à $\mathbf{j} \mathbf{2}$ d'un remplissage par sérum albumine concentrée à la dose de $1 \mathrm{~g} / \mathrm{kg}$ (maximum $100 \mathrm{~g} / \mathrm{j}$ ) associé à un arrêt du traitement diurétique

Absence d'état de choc hypovolémique ou septique nécessitant des substances vasopressives

Absence d'anomalie rénale définie par protéinurie, hématurie et échographie normales 
mauvais pronostic chez ces patients. Par conséquent, les anciennes définitions entraînaient possiblement une perte de chance chez ces patients [29,30]. La nouvelle classification d'AKI a donc été adoptée, et une définition unique pour le SHR-AKI est actuellement reconnue [28]. L'entité appelée antérieurement SHR de type 2 est de plus en plus assimilée à une maladie rénale chronique et non à une IRA du fait de son évolution lente [31].

\section{Prise en charge thérapeutique spécifique de l'insuffisance rénale aiguë chez le patient cirrhotique}

\section{AKI}

Devant toute IRA chez le patient cirrhotique, il est nécessaire de suspendre ou de limiter les traitements néphrotoxiques (les diurétiques, les AINS, les IEC, les antibiotiques), de corriger l'hypovolémie et de traiter un éventuel sepsis (Fig. 2). Le choix du soluté de remplissage dépend de la cause suspectée : les cristalloïdes sont à privilégier en cas de diarrhée ou de déshydratation sur diurétiques, les culots globulaires en cas d'hémorragie avec un objectif de maintien de l'hémoglobine entre 7 et $9 \mathrm{~g} / \mathrm{dl}$ et le sérum d'albumine concentrée à $20 \%$ (SAC) en cas de sepsis, de SHR suspecté ou en l'absence de cause évidente [9,27] dont la posologie d'administration est détaillée ci-après.

De nombreux patients sont traités par betabloquants dans l'indication de prévention primaire ou secondaire de l'hémorragie digestive sur hypertension portale (HTP). La responsabilité de ce traitement dans la survenue d'IRA chez le patient cirrhotique décompensé est actuellement discutée [32]. De fait, en cas d'IRA, il est recommandé d'arrêter le traitement par bêtabloquants et d'en réévaluer prudemment la nécessité de réintroduction [27].
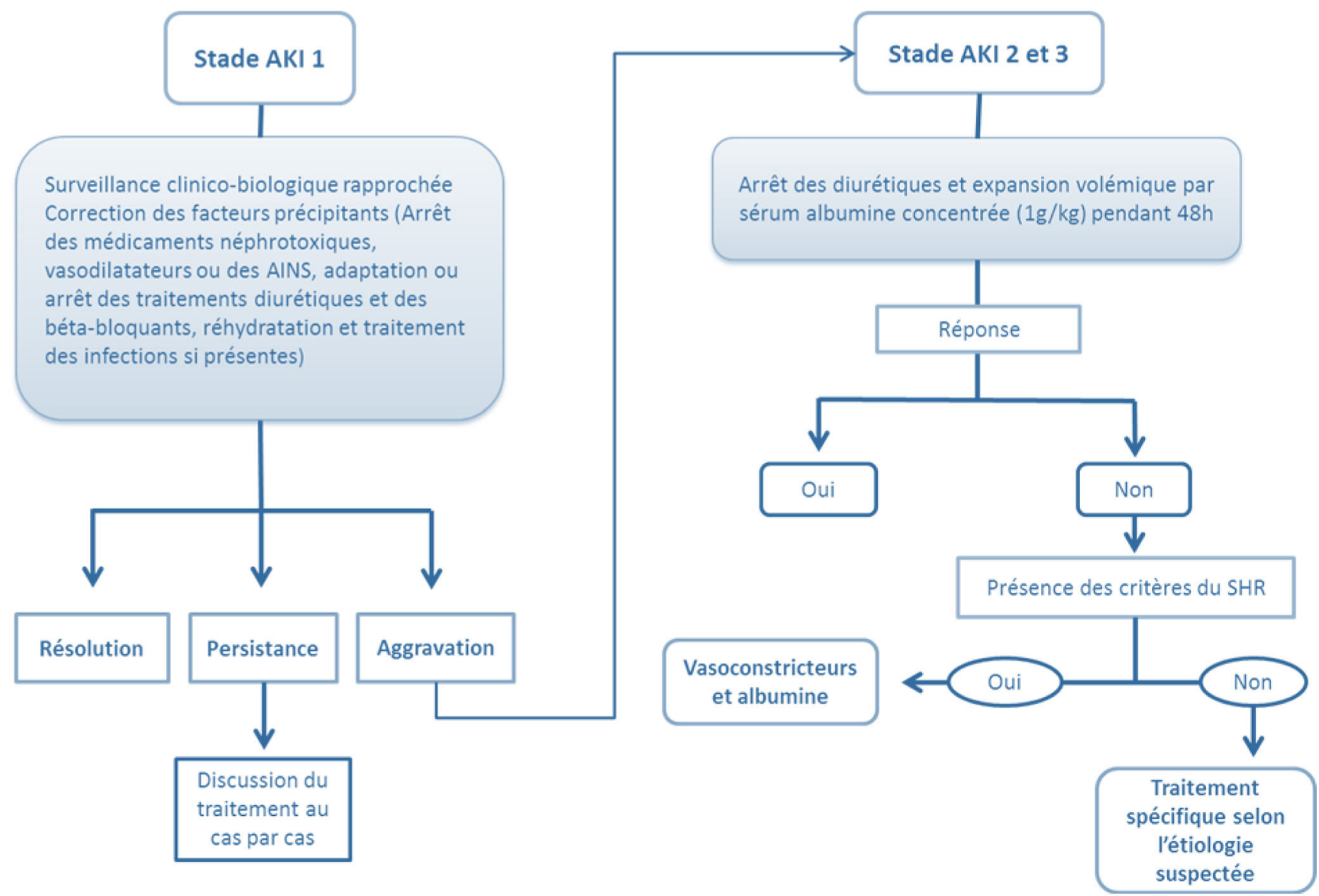

Réponse complète définie par un écart entre la créatininémie finale et basale $<26,5 \mu \mathrm{mol} / 1$.

Réponse partielle définie une amélioration du stade AKI et un écart entre la créatininémie finale et basale $\geq 26,5 \mu \mathrm{mol} / 1$.

Fig. 2 Algorithme de prise en charge proposé par l'EASL (adapté de Angeli et al. [28]) 
En cas d'AKI de stades 2 et 3, un SHR peut être évoqué. Ainsi, il est recommandé de réaliser une épreuve de remplissage standardisée par SAC à la dose de $1 \mathrm{~g} / \mathrm{kg}$ par jour pendant 48 heures (dose maximum de 100 g/j) [18,27]. En l'absence d'amélioration et si tous les critères diagnostiques sont présents, le diagnostic de SHR peut être retenu (Fig. 2).

\section{Syndrome hépatorénal}

En rapport avec la physiopathologie de ce syndrome, le traitement repose principalement sur un traitement médical combinant l'administration de SAC $(20-40 \mathrm{~g} / \mathrm{j})$ à un traitement vasoconstricteur administré par voie intraveineuse. Le remplissage vasculaire par SAC permet de lutter d'une part contre l'hypovolémie efficace et d'autre part d'améliorer la contractilité cardiaque [33] alors que le traitement vasoconstricteur permet de réduire la vasodilatation splanchnique [34-36].

La principale substance vasoconstrictrice utilisée est la terlipressine. Elle peut être administrée par bolus intraveineux $(0,5$ à $1 \mathrm{mg} \times 4-6 / \mathrm{j}$ jusqu'à $2 \mathrm{mg} \times 4-6 / \mathrm{j})$ ou en perfusion continue avec une dose de départ de $2 \mathrm{mg} / 24 \mathrm{~h}$ pouvant être majorée jusqu'à $12 \mathrm{mg} / \mathrm{j}$ en cas d'absence de réponse $[30,37,38]$. La terlipressine expose à plusieurs effets secondaires pouvant mener à l'interruption du traitement. Parmi les plus fréquents ou sévères, nous pouvons citer la diarrhée [37] ou des événements cardiovasculaires tels qu'une ischémie mésentérique ou un œdème aigu pulmonaire (OAP) [37,39]. Une étude a montré une meilleure tolérance sans perte d'efficacité de la perfusion continue par rapport à l'administration par bolus pouvant faire préférer cette voie d'administration actuellement [40]. Trois suspicions d'ischémie mésentérique, cinq OAP et deux cas de diarrhée ont été recensés dans le groupe bolus contre seulement deux OAP dans le groupe perfusion continue. Au total, le pourcentage d'effets secondaires rapporté dans les deux groupes était respectivement de 43,2 et 20,6\% $(p<0,05)$. Cette diminution était probablement liée à une baisse de la posologie efficace de terlipressine utilisée dont la médiane était de $3,5 \mathrm{mg} / \mathrm{j}$ dans le groupe bolus et de $2,2 \mathrm{mg} / \mathrm{j}$ dans le groupe perfusion continue $(p=0,0001)$ [40].

La noradrénaline peut également être utilisée comme traitement vasoconstricteur notamment en cas de contreindication à la terlipressine. Plusieurs études ont montré une efficacité à la dose de $0,5 \mathrm{mg} / \mathrm{h}$ jusqu'à $3 \mathrm{mg} / \mathrm{h}$ associée à l'utilisation d'albumine [41-43]. La posologie de la noradrénaline était augmentée progressivement pour atteindre une majoration de la pression artérielle moyenne de $10 \mathrm{mmHg}$ ou une augmentation de la diurèse à plus de $200 \mathrm{ml}$ [41-43]. Plusieurs études ont comparé l'efficacité de la noradrénaline à la terlipressine dans le SHR. Aucune différence n'avait été montrée jusqu'à récemment en termes de mortalité et de réponse au traitement entre ces deux molécules [44]. Une étude randomisée contrôlée en protocole ouvert récente a comparé la noradrénaline et la terlipressine chez des patients cirrhotiques présentant un SHR en contexte d'ACLF. Dans cette étude comprenant 120 patients, la posologie médiane de terlipressine était de $2,02 \mathrm{mg} / \mathrm{j}$ et de NAD de $1,11 \mathrm{mg} / \mathrm{h}$. Une réponse complète était obtenue respectivement dans 40 et $16,7 \%$ des cas au $14^{\mathrm{e}}$ jour. La mortalité à 28 jours était de 51,7 contre $80 \%(p=0,001)$ [26]. Une étude indienne de 60 patients de moins bonne qualité méthodologique ne montrait aucune différence significative entre les deux molécules [45]. À la lumière de ces récents travaux, les experts de l'AFEF et de la SFAR ont recommandé l'utilisation de la terlipressine en perfusion continue en première intention dans le traitement du SHR (Recommandation 5.2, Grade 2+, accord fort) [38].

Pour information, l'association midodrine-octreotide (de $7,5 \mathrm{mg} / 8 \mathrm{~h}$ à $12,5 \mathrm{mg} / 8 \mathrm{~h}$ de midodrine et $100 \mu \mathrm{g} / 8 \mathrm{~h}$ à $200 \mu \mathrm{g} / 8 \mathrm{~h}$ d'octréotide) a été proposée dans les pays ne disposant pas de la terlipressine. Cette association est toutefois moins efficace que le traitement de référence et n'est donc pas à proposer en première intention [46].

L'efficacité du traitement est évaluée à 48 heures où la réponse est définie par une baisse de $25 \%$ du taux de créatininémie. Le traitement sera poursuivi jusqu'à l'obtention d'une créatininémie inférieure à $133 \mu \mathrm{mol} / \mathrm{l}$ (réponse complète) ou sera poursuivi au maximum pendant 14 jours en l'absence de réponse ou de réponse partielle (baisse d'au moins $50 \%$ de la créatininémie de base, mais sans atteindre l'objectif de créatininémie inférieure à $133 \mu \mathrm{mol} / \mathrm{l}$ ) [27]. En l'absence de réponse, la posologie du traitement peut être majorée par paliers. Par exemple, la terlipressine peut être majorée de $2 \mathrm{mg} / 24 \mathrm{~h}$ à $4 \mathrm{mg} / 24 \mathrm{~h}$ en perfusion continue (et ce jusqu'à $12 \mathrm{mg} / 24 \mathrm{~h}$ ) en l'absence de réponse à 48 heures.

Le taux de réponse complète pour le traitement médical du SHR par albumine/terlipressine est de 20 à $56 \%$ selon les études $[26,29,40,47]$. Chez les patients répondeurs, $20 \%$ présenteront une récidive du SHR à l'arrêt du traitement, mais une majorité répondra à une reprise de celui-ci [48]. Le pronostic à court terme est variable selon les études, mais est meilleur chez les patients répondeurs au traitement médical $[26,40,47]$ avec par exemple une survie à trois mois de 70 contre $41 \%$ dans l'étude de Cavallin et al. Cependant, même en cas de réponse complète, la mortalité reste élevée du fait de la sévérité de la cirrhose sous-jacente sur laquelle le traitement du SHR n'a pas d'impact. En conséquence, indépendamment de la réponse au traitement médical, la survenue d'un SHR devra faire discuter le transfert du patient vers un centre de $\mathrm{TH}$.

\section{Épuration extrarénale (EER) et AKI}

Le pronostic des patients cirrhotiques présentant une AKI et nécessitant une EER est mauvais (particulièrement pour les 
patients non éligibles à la TH). La mortalité rapportée varie entre 73 et $85 \%$ et ne semble pas différente entre les diverses causes d'AKI [49-52].

Malgré un pronostic très péjoratif, les experts de l'AFEF et de la SFAR ont estimé que l'EER dans le cadre d'une AKI chez un patient cirrhotique n'est pas contre-indiquée à condition que celle-ci soit de courte durée et intégrée dans un projet thérapeutique tel qu'une TH et/ou dans le contexte d'un facteur déclenchant réversible sous traitement comme un sepsis (Recommandation 5.1, avis d'experts) [38].

Les indications d'EER sont les mêmes que pour les autres causes d'IRA dans la population générale et principalement en cas de signe de gravité : acidose métabolique sévère, hyperkaliémie menaçante, anasarque avec OAP [27].

Peu d'études comparant les techniques d'EER (continue ou intermittente) dans cette population sont disponibles. Dans les études de Staufer et al. et de Lenhart et al., la mortalité plus élevée observée chez les patients bénéficiant d'une EER continue $[52,53]$ pourrait être expliquée par une gravité plus importante des malades bénéficiant d'EER continue (souvent privilégiée pour sa meilleure tolérance hémodynamique) [52]. À ce jour et en l'absence d'essai contrôlé dédié, les experts internationaux s'accordent sur une préférence d'utilisation d'une technique d'EER continue [54] même si ce choix doit dépendre aussi de l'expertise locale du centre.

L'espace-temps idéal pour l'initiation de l'EER n'a pas été bien étudié chez les patients cirrhotiques. Les données de la littérature chez des patients ne présentant pas d'hépatopathie chronique suggèrent que le maintien d'une balance hydrosodée négative pourrait améliorer le pronostic sans argument pour un bénéfice supérieur en cas d'initiation précoce de l'EER [54-56].

En cas de projet de TH, l'EER peut être envisagée comme technique d'attente/de pont vers la transplantation. Concernant le SHR après $\mathrm{TH}$, le taux de régression rapporté varie entre 58 et $90 \%$ selon les études [57-60]. L'absence de normalisation de la fonction rénale pourrait être liée à des lésions tubulaires rénales en lien avec une ischémie rénale prolongée secondaire au SHR [57,61]. En cas de NTA, la récupération de la fonction rénale est évaluée à $71 \%$ à trois mois [62]. Une EER de durée prolongée avant transplantation est un facteur prédictif d'absence de normalisation de la fonction rénale après transplantation [57]. De nombreuses données de la littérature suggèrent un impact péjoratif d'un épisode d'AKI sur la fonction rénale à long terme [63-66]. De fait, la présence d'un SHR ne répondant pas au traitement médical et/ou à la nécessité d'une EER n'est pas un frein à un projet de $\mathrm{TH}$, mais a un impact probable sur la fonction rénale après transplantation.

En l'absence de projet de TH, l'instauration d'une EER dans un contexte de résistance au traitement conventionnel reste controversée, car n'ayant pas démontré d'amélioration de la survie sans transplantation $[50,54]$. Les études actuelles n'ont pas permis d'identifier un sous-groupe de patients pouvant tirer bénéfice de l'EER en termes de survie globale [67]. Si une EER est indiquée, elle pourra être discutée au cas par cas selon le pronostic du patient évalué par un score de défaillance d'organes (détaillé dans le paragraphe suivant) et d'autres paramètres (réversibilité de la pathologie rénale, âge, comorbidités, volonté du patient) [68].

\section{Acute-on-chronic liver failure (ACLF) et AKI}

La survenue d'une AKI chez un patient cirrhotique décompensé est observée le plus souvent au cours d'une dégradation brutale de la fonction hépatocellulaire s'inscrivant dans un contexte d'ACLF. La survenue d'un ACLF est le plus souvent secondaire à un événement précipitant et est associée au développement de défaillances d'organes extrahépatiques. Il est associé à une mortalité élevée à 28 jours (33\%) et à trois mois (51\%) [21]. Le score CLIF-SOFA définissant les différentes défaillances d'organes, et développé sur le modèle du score SOFA, a été validé comme score prédictif de mortalité dans cette population (Tableau 3). La classification ACLF dépendante du nombre de défaillances d'organes définies par ce score identifie quatre stades de pronostics différents à court terme (Tableau 4). Au cours de l'ACLF, la défaillance rénale est la défaillance la plus observée $(56 \%$ de tous les patients avec ACLF ; $59 \%$ pour ACLF 1; $45 \%$ pour ACLF 2 ; $70 \%$ pour ACLF 3) [21].

$\mathrm{Au}$ cours de la prise en charge en réanimation, le pronostic du patient peut être évalué par le nombre de défaillances d'organes à l'aide de cette classification ou par un autre score de défaillances d'organes calculé idéalement entre l'admission et le $15^{\mathrm{e}}$ jour $[69,70]$. Ces scores sont en effet supérieurs dans la prédiction de la mortalité des patients cirrhotiques en réanimation comparés aux scores MELD et de Child-Pugh [71,72], et leur calcul séquentiel permet d'affiner l'évaluation pronostique des patients. À titre d'exemple, la survie sans

Tableau 3 Définition des défaillances d'organes selon le score CLIF-SOFA [21]

\begin{tabular}{|c|c|}
\hline Organe/système & Définition de la défaillance \\
\hline Foie & Bilirubine $\geq 205 \mu \mathrm{mol} / \mathrm{l}$ \\
\hline Rein & $\begin{array}{l}\text { Créatininémie } \geq 177 \mu \mathrm{mol} / \mathrm{l} \\
\text { ou épuration extrarénale }\end{array}$ \\
\hline Cérébral/EH & Grades 3-4 \\
\hline Coagulation & INR $\geq 2,5$ ou plaquettes $\leq 20 \mathrm{~g} / \mathrm{l}$ \\
\hline Circulatoire & $\begin{array}{l}\text { Amines vasopressives (au moins } \\
\text { une parmi dopamine, dobutamine, } \\
\text { épinéphrine et norépinéphrine) } \\
\text { ou terlipressine }\end{array}$ \\
\hline \multirow[t]{2}{*}{ Poumon } & $\mathrm{PaO}_{2} / \mathrm{FiO}_{2} \leq 200$ \\
\hline & $\mathrm{SpO}_{2} / \mathrm{FiO}_{2} \leq 214$ \\
\hline
\end{tabular}




\begin{tabular}{|c|c|c|c|}
\hline $\begin{array}{l}\text { Stade } \\
\text { ACLF }\end{array}$ & Définition & $\begin{array}{l}\text { Mortalité à } \\
28 \text { jours (\%) }\end{array}$ & $\begin{array}{l}\text { Mortalité à } \\
90 \text { jours (\%) }\end{array}$ \\
\hline ACLF 0 & $\begin{array}{l}\text { Absence de défaillance d'organes } \\
o u \\
\text { Une défaillance d'organes non rénale associée à une créatininémie inférieure à } \\
133 \mu \mathrm{mol} / 1 \text { et sans encéphalopathie hépatique } \\
o u \\
\text { Défaillance neurologique et créatininémie }<133 \mu \mathrm{mol} / 1\end{array}$ & 4,7 & 14 \\
\hline ACLF 1 & $\begin{array}{l}\text { Créatininémie } \geq 177 \mu \mathrm{mol} / 1 \\
\text { ou } \\
\text { Défaillance d'organes non rénale associée à une créatininémie entre } 133 \text { et } 168 \mu \mathrm{mol} / 1 \\
\text { ou } \\
\text { Défaillance neurologique isolée associée à une créatininémie entre } 133 \text { et } 168 \mu \mathrm{mol} / 1\end{array}$ & 22,1 & 40,7 \\
\hline ACLF 2 & Deux défaillances d'organes & 32 & 52,3 \\
\hline ACLF 3 & Trois défaillances d'organes & 76,7 & 79,1 \\
\hline
\end{tabular}

transplantation des patients ACLF de grade 2 à l'admission est d'environ 60 contre $40 \%$ si ce grade persiste entre $\mathrm{j} 3$ et $\mathrm{j} 7$.

Comme évoqué précédemment, la décision d'instauration de l'EER doit être discutée au cas par cas principalement selon la perspective d'une $\mathrm{TH}$ à court terme. L'éligibilité à la TH dépend principalement de l'âge, des comorbidités (cardiologique, respiratoire, néoplasique), de critères addictologiques, mais aussi de la sévérité des défaillances d'organes. Les critères stricts d'éligibilité à la TH sont susceptibles de varier entre les équipes de transplantation et dans le temps au sein de la même équipe [73] et ne peuvent être donc généralisés.

Plusieurs études ont rapporté une survie après transplantation en contexte d'ACLF supérieure à $80 \%$ à un an et peu différente des patients ne présentant pas d'ACLF [74-76]. Après TH, la défaillance rénale au jour de la TH était associée à un meilleur pronostic que la présence d'une défaillance circulatoire ou respiratoire [76]. En contexte d'ACLF, une AKI nécessitant ou non un recours à l'EER ne constitue donc pas un obstacle à la $\mathrm{TH}$ à lui seul.

En l'absence de projet de TH, le pronostic évalué par le calcul séquentiel du grade ACLF et le potentiel de réversibilité de la pathologie rénale évaluée de manière multidisciplinaire avec le néphrologue référent doivent être pris en compte pour décider ou non de l'instauration d'une EER $[9,54]$. Enfin, d'autres paramètres doivent être évoqués tels que l'âge, la présence d'autres comorbidités et la volonté du patient [68].

\section{Conclusion}

L'IRA ou AKI est une complication fréquente chez le patient cirrhotique dont la classification a été révisée récemment. Le SHR est une des causes fréquentes d'AKI et survient princi- palement chez le patient cirrhotique décompensé dont la prise en charge thérapeutique de référence associe un traitement vasoconstricteur à la perfusion d'albumine concentrée. La classification ACLF, dont les stades sont définis par le nombre de défaillances d'organes, permet de mieux appréhender le pronostic de ces patients. La décision d'une EER dépend principalement de l'existence d'un projet de TH à court terme. En l'absence d'un tel projet, la décision d'EER doit être prise au cas par cas selon le pronostic du patient évalué par le nombre de défaillances d'organes (grade ACLF).

Liens d'intérêts : les auteurs déclarent ne pas avoir de lien d'intérêt.

\section{Références}

1. Cirrhose [Internet]. Inserm. [cited 2018 Jul 21]. Available from: https://www.inserm.fr/information-en-sante/dossiers-information/ cirrhose

2. Piton G, Chaignat C, Giabicani M, Cervoni JP, Tamion F, Weiss E, Paugam-Burtz C, Capellier G, Di Martino V, (2016) Prognosis of cirrhotic patients admitted to the general ICU. Ann Intensive Care 6: 94

3. McPhail MJW, Parrott F, Wendon JA, Harrison DA, Rowan KA, Bernal W, (2018) Incidence and outcomes for patients with cirrhosis admitted to the United Kingdom critical care units. Crit Care Med 6: 705-712

4. O'Brien AJ, Welch CA, Singer M, Harrison DA, (2012) Prevalence and outcome of cirrhosis patients admitted to UK intensive care: a comparison against dialysis-dependent chronic renal failure patients. Intensive Care Med 38: 991-1000

5. McPhail MJW, Shawcross DL, Abeles RD, Chang A, Patel V, Lee GH, Abdulla M, Sizer E, Willars C, Auzinger G, Bernal W, Wendon JA, (2015) Increased survival for patients with cirrhosis and organ failure in liver intensive care and validation of the chronic Liver Failure-Sequential Organ Failure Scoring System. Clin Gastroenterol Hepatol 13: 1353-1360.e8 
6. Carbonell N, Pauwels A, Serfaty L, Fourdan O, Lévy VG, Poupon R, (2004) Improved survival after variceal bleeding in patients with cirrhosis over the past two decades. Hepatology 40: 652-659

7. de Franchis R, Baveno V Faculty, (2010) Revising consensus in portal hypertension: report of the Baveno $\mathrm{V}$ consensus workshop on methodology of diagnosis and therapy in portal hypertension. J Hepatol 53: 762-768

8. de Franchis R, Baveno VI Faculty, (2015) Expanding consensus in portal hypertension: report of the Baveno VI Consensus Workshop: Stratifying risk and individualizing care for portal hypertension. J Hepatol 63: 743-752

9. Nadim MK, Durand F, Kellum JA, Levitsky J, O'Leary JG, Karvellas CJ, Bajaj JS, Davenport A, Jalan R, Angeli P, Caldwell $\mathrm{SH}$, Fernández J, Francoz C, Garcia-Tsao G, Ginès P, Ison MG, Kramer DJ, Mehta RL, Moreau R, Mulligan D, Olson JC, Pomfret EA, Senzolo M, Steadman RH, Subramanian RM, Vincent JL, Genyk YS, (2016) Management of the critically ill patient with cirrhosis: a multidisciplinary perspective. J Hepatol 64: 717-735

10. Brower RG, Lanken PN, MacIntyre N, Matthay MA, Morris A, Ancukiewicz M, Schoenfeld D, Thompson BT; National Heart ; Lung, and Blood Institute ARDS Clinical Trials Network, (2004) Higher versus lower positive end-expiratory pressures in patients with the acute respiratory distress syndrome. N Engl J Med 351: 327-336

11. Rivers E, Nguyen B, Havstad S, Ressler J, Muzzin A, Knoblich B, Peterson E, Tomlanovich M; Early Goal-Directed Therapy Collaborative Group, (2001) Early goal-directed therapy in the treatment of severe sepsis and septic shock. N Engl J Med 345: $1368-1377$

12. Garcia-Tsao G, Parikh CR, Viola A, (2008) Acute kidney injury in cirrhosis. Hepatology 48: 2064-2077

13. Kamath PS, Wiesner RH, Malinchoc M, Kremers W, Therneau TM, Kosberg CL, D'Amico G, Dickson ER, Kim WR, (2001) A model to predict survival in patients with end-stage liver disease. Hepatology 33: 464-470

14. Wiesner RH, McDiarmid SV, Kamath PS, Edwards EB, Malinchoc M, Kremers WK, Krom RA, Kim WR, (2001) MELD and PELD: application of survival models to liver allocation. Liver Transplant 7: 567-580

15. Wiesner R, Edwards E, Freeman R, Harper A, Kim R, Kamath P, Kremers W, Lake J, Howard T, Merion RM, Wolfe RA, Krom R; United Network for Organ Sharing Liver Disease Severity Score Committee, (2003) Model for end-stage liver disease (MELD) and allocation of donor livers. Gastroenterology 124: 91-96

16. Angeli P, Ginès P, Wong F, Bernardi M, Boyer TD, Gerbes A, Moreau R, Jalan R, Sarin SK, Piano S, Moore K, Lee SS, Durand F, Salerno F, Caraceni P, Kim WR, Arroyo V, Garcia-Tsao G, (2015) Diagnosis and management of acute kidney injury in patients with cirrhosis: revised consensus recommendations of the International Club of Ascites. J Hepatol 62: 968-974

17. Khwaja A, (2012) KDIGO clinical practice guidelines for acute kidney injury. Nephron Clin Pract 120: c179-c184

18. Wong F, Angeli P, (2017) New diagnostic criteria and management of acute kidney injury. J Hepatol 66: 860-861

19. Arroyo V, Terra $C$, Ginès $P$, (2007) Advances in the pathogenesis and treatment of type-1 and type-2 hepatorenal syndrome. J Hepatol 46: 935-946

20. Piano S, Tonon M, Angeli P, (2018) Management of ascites and hepatorenal syndrome. Hepatol Int 12: 122-134

21. Moreau R, Jalan R, Gines P, Pavesi M, Angeli P, Cordoba J, Durand F, Gustot T, Saliba F, Domenicali M, Gerbes A, Wendon J, Alessandria C, Laleman W, Zeuzem S, Trebicka J, Bernardi M, Arroyo V; CANONIC Study Investigators of the EASL-CLIF Consortium, (2013) Acute-on-chronic liver failure is a distinct syndrome that develops in patients with acute decompensation of cirrhosis. Gastroenterology 144: 1426-1437, 1437.e1-9

22. Huelin P, Piano S, Solà E, Stanco M, Solé C, Moreira R, Pose E, Fasolato S, Fabrellas N, de Prada G, Pilutti C, Graupera I, Ariza X, Romano A, Elia C, Cárdenas A, Fernández J, Angeli P, Ginès P, (2017) Validation of a Staging System for Acute Kidney Injury in Patients With Cirrhosis and Association With Acute-on-Chronic Liver Failure. Clin Gastroenterol Hepatol 15: 438-445.e5

23. Fagundes C, Barreto R, Guevara M, Garcia E, Solà E, Rodríguez E, Graupera I, Ariza X, Pereira G, Alfaro I, Cárdenas A, Fernández J, Poch E, Ginès P, (2013) A modified acute kidney injury classification for diagnosis and risk stratification of impairment of kidney function in cirrhosis. J Hepatol 59: 474-481

24. Ginès $\mathrm{P}$, Solà $\mathrm{E}$, Angeli $\mathrm{P}$, Wong $\mathrm{F}$, Nadim $\mathrm{MK}$, Kamath $\mathrm{P}$, (2018) Hepatorenal syndrome. Nat Rev Dis Primer 4: 23

25. Martín-Llahí M, Guevara M, Torre A, Fagundes C, Restuccia T, Gilabert R, Solá E, Pereira G, Marinelli M, Pavesi M, Fernández J, Rodés J, Arroyo V, Ginès P, (2011) Prognostic importance of the cause of renal failure in patients with cirrhosis. Gastroenterology 140: 488-496.e4

26. Arora V, Maiwall R, Vijayaraghavan R, Jindal A, Saggere Muralikrishna S, Kumar G, Jain P, Sarin SK, (2018) Terlipressin is superior to noradrenaline in the management of acute kidney injury in acute on chronic liver failure. Hepatology (doi: 10.1002/hep.30208. [Epub ahead of print])

27. European Association for the Study of the Liver. Electronic address: easloffice@easloffice.eu; European Association for the Study of the Liver, (2018) EASL Clinical Practice Guidelines for the management of patients with decompensated cirrhosis. J Hepatol 69: 406-460

28. Angeli P, Gines P, Wong F, Bernardi M, Boyer TD, Gerbes A, Moreau R, Jalan R, Sarin SK, Piano S, Moore K, Lee SS, Durand F, Salerno F, Caraceni P, Kim WR, Arroyo V, Garcia-Tsao G, (2015) Diagnosis and management of acute kidney injury in patients with cirrhosis: revised consensus recommendations of the International Club of Ascites. Gut 64: 531-537

29. Rodríguez E, Elia C, Solà E, Barreto R, Graupera I, Andrealli A, Pereira G, Poca M, Sánchez J, Guevara M, Soriano G, Alessandria C, Fernández J, Arroyo V, Ginès P, (2014) Terlipressin and albumin for type-1 hepatorenal syndrome associated with sepsis. J Hepatol 60: 955-961

30. Boyer TD, Sanyal AJ, Garcia-Tsao G, Blei A, Carl D, Bexon AS, Teuber P; Terlipressin Study Group, (2011) Predictors of response to terlipressin plus albumin in hepatorenal syndrome (HRS) type 1: relationship of serum creatinine to hemodynamics. J Hepatol 55: 315-321

31. Wong F, Nadim MK, Kellum JA, Salerno F, Bellomo R, Gerbes A, Angeli P, Moreau R, Davenport A, Jalan R, Ronco C, Genyk Y, Arroyo V, (2011) Working Party proposal for a revised classification system of renal dysfunction in patients with cirrhosis. Gut 60: 702-709

32. Tripathi D, Hayes PC, (2014) Beta-blockers in portal hypertension: new developments and controversies. Liver Int 34: 655-667

33. Arroyo V, García-Martinez R, Salvatella X, (2014) Human serum albumin, systemic inflammation, and cirrhosis. J Hepatol 61: 396-407

34. Hadengue A, Gadano A, Moreau R, Giostra E, Durand F, Valla D, Erlinger S, Lebrec D, (1998) Beneficial effects of the 2-day administration of terlipressin in patients with cirrhosis and hepatorenal syndrome. J Hepatol 29: 565-570

35. Lenz K, Hörtnagl H, Druml W, Reither H, Schmid R, Schneeweiss B, Laggner A, Grimm G, Gerbes AL, (1991) Ornipressin in the treatment of functional renal failure in decompensated liver cirrhosis. Effects on renal hemodynamics and atrial natriuretic factor. Gastroenterology 101: 1060-7 
36. Valla D, Lee SS, Moreau R, Hadengue A, Sayegh R, Lebrec D, (1985) Effects of glypressin on the splanchnic and systemic circulation in patients with cirrhosis. Gastroenterol Clin Biol 9: 877-880

37. Sanyal AJ, Boyer T, Garcia-Tsao G, Regenstein F, Rossaro L, Appenrodt B, Blei A, Gülberg V, Sigal S, Teuber P; Terlipressin Study Group, (2008) A randomized, prospective, double-blind, placebo-controlled trial of terlipressin for type 1 hepatorenal syndrome. Gastroenterology 134: 1360-8

38. Paugam-Burtz C, Levesque E, Louvet A, Thabut D, Amathieu R, Bureau C, Camus C, Chanques G, Faure S, Ferrandière M, Fran$\operatorname{coz} \mathrm{C}$, Galbois A, Gustot T, Ichai C, Ichai P, Jaber S, Lescot T, Moreau R, Roullet S, Saliba F, Thevenot T, Velly L, Weiss E, (2018) Liver failure in ICU. https://sfar.org/wp-content/uploads/ 2018/09/RFE-IH-soins-critiques.pdf

39. Martín-Llahí M, Pépin MN, Guevara M, Díaz F, Torre A, Monescillo A, Soriano G, Terra C, Fábrega E, Arroyo V, Rodés J, Ginès P; TAHRS Investigators, (2008) Terlipressin and albumin vs albumin in patients with cirrhosis and hepatorenal syndrome: a randomized study. Gastroenterology 134: 1352-1359

40. Cavallin M, Piano S, Romano A, Fasolato S, Frigo AC, Benetti G, Gola E, Morando F, Stanco M, Rosi S, Sticca A, Cillo U, Angeli P, (2016) Terlipressin given by continuous intravenous infusion versus intravenous boluses in the treatment of hepatorenal syndrome: a randomized controlled study. Hepatology 63: 983-992

41. Alessandria C, Ottobrelli A, Debernardi-Venon W, Todros L, Cerenzia MT, Martini S, Balzola F, Morgando A, Rizzetto M, Marzano A, (2007) Noradrenalin vs terlipressin in patients with hepatorenal syndrome: a prospective, randomized, unblinded, pilot study. J Hepatol 47: 499-505

42. Singh V, Ghosh S, Singh B, Kumar P, Sharma N, Bhalla A, Sharma AK, Choudhary NS, Chawla Y, Nain CK, (2012) Noradrenaline vs. terlipressin in the treatment of hepatorenal syndrome: a randomized study. J Hepatol 56: 1293-1298

43. Sharma P, Kumar A, Shrama BC, Sarin SK, (2008) An open label, pilot, randomized controlled trial of noradrenaline versus terlipressin in the treatment of type 1 hepatorenal syndrome and predictors of response. Am J Gastroenterol 103: 1689-1697

44. Facciorusso A, Chandar AK, Murad MH, Prokop LJ, Muscatiello N, Kamath PS, Singh S, (2017) Comparative efficacy of pharmacological strategies for management of type 1 hepatorenal syndrome: a systematic review and network meta-analysis. Lancet Gastroenterol Hepatol 2: 94-102

45. Saif RU, Dar HA, Sofi SM, Andrabi MS, Javid G, Zargar SA, (2018) Noradrenaline versus terlipressin in the management of type 1 hepatorenal syndrome: A randomized controlled study. Indian J Gastroenterol 37: 424-429 (doi: 10.1007/s12664-0180876-3. Epub 2018 Sep 3)

46. Cavallin M, Kamath PS, Merli M, Fasolato S, Toniutto P, Salerno F, Bernardi M, Romanelli RG, Colletta C, Salinas F, Di Giacomo A, Ridola L, Fornasiere E, Caraceni P, Morando F, Piano S, Gatta A, Angeli P; Italian Association for the Study of the Liver Study Group on Hepatorenal Syndrome, (2015) Terlipressin plus albumin versus midodrine and octreotide plus albumin in the treatment of hepatorenal syndrome: a randomized trial. Hepatology 62: $567-574$

47. Boyer TD, Sanyal AJ, Wong F, Frederick RT, Lake JR, O'Leary JG, Ganger D, Jamil K, Pappas SC; REVERSE Study Investigators, (2016) Terlipressin plus albumin is more effective than albumin alone in improving renal function in patients with cirrhosis and hepatorenal syndrome type 1. Gastroenterology 150: 1579-1589.e2

48. Salerno F, Gerbes A, Ginès P, Wong F, Arroyo V, (2007) Diagnosis, prevention and treatment of hepatorenal syndrome in cirrhosis. Gut 56: 1310-1318
49. Allegretti AS, Parada XV, Eneanya ND, Gilligan H, Xu D, Zhao S, Dienstag JL, Chung RT, Thadhani RI, (2018) Prognosis of Patients with Cirrhosis and AKI Who Initiate RRT. Clin J Am Soc Nephrol CJASN 13: 16-25

50. Zhang Z, Maddukuri G, Jaipaul N, Cai CX, (2015) Role of renal replacement therapy in patients with type 1 hepatorenal syndrome receiving combination treatment of vasoconstrictor plus albumin. J Crit Care 30: 969-974

51. Sourianarayanane A, Raina R, Garg G, McCullough AJ, O'Shea RS, (2014) Management and outcome in hepatorenal syndrome: need for renal replacement therapy in non-transplanted patients. Int Urol Nephrol 46: 793-800

52. Staufer K, Roedl K, Kivaranovic D, Drolz A, Horvatits T, Rasoul-Rockenschaub S, Zauner C, Trauner M, Fuhrmann V, (2017) Renal replacement therapy in critically ill liver cirrhotic patients-outcome and clinical implications. Liver Int 37: 843-850

53. Lenhart A, Hussain S, Salgia R, (2018) Chances of renal recovery or liver transplantation after hospitalization for alcoholic liver disease requiring dialysis. Dig Dis Sci 63: 2800-2809 (doi: 10.1007/s10620-018-5170-9. Epub 2018 Jun 22)

54. Durand F, Olson JC, Nadim MK, (2017) Renal dysfunction and cirrhosis. Curr Opin Crit Care 23: 457-462

55. Gaudry S, Hajage D, Dreyfuss D, (2016) Initiation of RenalReplacement Therapy in the Intensive Care Unit. N Engl J Med 375: 1901-1902

56. Vaara ST, Korhonen A-M, Kaukonen KM, Nisula S, Inkinen O, Hoppu S, Laurila JJ, Mildh L, Reinikainen M, Lund V, Parviainen I, Pettilä V; FINNAKI Study Group, (2012) Fluid overload is associated with an increased risk for 90 -day mortality in critically ill patients with renal replacement therapy: data from the prospective FINNAKI study. Crit Care Lond Engl 16: R197

57. Wong F, Leung W, Al Beshir M, Marquez M, Renner EL, (2015) Outcomes of patients with cirrhosis and hepatorenal syndrome type 1 treated with liver transplantation. Liver Transplant 21: 300-307

58. Marik PE, Wood K, Starzl TE, (2006) The course of type 1 hepato-renal syndrome post liver transplantation. Nephrol Dial Transplant 21: 478-82

59. Gonwa TA, Morris CA, Goldstein RM, Husberg BS, Klintmalm GB, (1991) Long-term survival and renal function following liver transplantation in patients with and without hepatorenal syndrome - experience in 300 patients. Transplantation 51: 428-430

60. Xu X, Ling Q, Zhang M, Gao F, He Z, You J, Zheng S, (2009) Outcome of patients with hepatorenal syndrome type 1 after liver transplantation: Hangzhou experience. Transplantation 87: $1514-1519$

61. Lameire NH, Vanholder R, (2004) Pathophysiology of ischaemic acute renal failure. Best Pract Res Clin Anaesthesiol 18: 21-36

62. Nadim MK, Genyk YS, Tokin C, Fieber J, Ananthapanyasut W, Ye W, Selby R, (2012) Impact of the etiology of acute kidney injury on outcomes following liver transplantation: acute tubular necrosis versus hepatorenal syndrome. Liver Transplant 18: 539-548

63. Bahirwani R, Forde KA, Mu Y, Lin F, Reese P, Goldberg D, Abt P, Reddy KR, Levine M, (2014) End-stage renal disease after liver transplantation in patients with pre-transplant chronic kidney disease. Clin Transplant 28: 205-210

64. Fabrizi F, Dixit V, Martin P, Messa P, (2011) Pre-transplant kidney function predicts chronic kidney disease after liver transplant: metaanalysis of observational studies. Dig Dis Sci 56: 1282-1289

65. Garces G, Contreras G, Carvalho D, Jaraba IM, Carvalho C, Tzakis A, Moon J, Ratnam K, Roth D, (2011) Chronic kidney disease after orthotopic liver transplantation in recipients receiving tacrolimus. Clin Nephrol 75: 150-157

66. Chawla LS, Eggers PW, Star RA, Kimmel PL, (2014) Acute kidney injury and chronic kidney disease as interconnected syndromes. N Engl J Med 371: 58-66 
67. Reverter E, Escorsell A, Fernández J, (2017) Renal replacement therapy in critically ill cirrhotic patients: a challenging balance between efficacy and futility. Liver Int 37: 817-819

68. McPhail MJW, Auzinger G, Bernal W, Wendon JA, (2016) Decisions on futility in patients with cirrhosis and organ failure. Hepatology 64: 986

69. Gustot T, Fernandez J, Garcia E, Morando F, Caraceni P, Alessandria C, Laleman W, Trebicka J, Elkrief L, Hopf C, SolísMunoz P, Saliba F, Zeuzem S, Albillos A, Benten D, MonteroAlvarez JL, Chivas MT, Concepción M, Córdoba J, McCormick A, Stauber R, Vogel W, de Gottardi A, Welzel TM, Domenicali M, Risso A, Wendon J, Deulofeu C, Angeli P, Durand F, Pavesi M, Gerbes A, Jalan R, Moreau R, Ginés P, Bernardi M, Arroyo $\mathrm{V}$; CANONIC Study Investigators of the EASL-CLIF Consortium, (2015) Clinical Course of acute-on-chronic liver failure syndrome and effects on prognosis. Hepatology 62: 243-252

70. Levesque E, Hoti E, Azoulay D, Ichaï P, Habouchi H, Castaing D, Samuel D, Saliba F, (2012) Prospective evaluation of the prognostic scores for cirrhotic patients admitted to an intensive care unit. J Hepatol 56: 95-102

71. Wehler M, Kokoska J, Reulbach U, Hahn EG, Strauss R, (2001) Short-term prognosis in critically ill patients with cirrhosis assessed by prognostic scoring systems. Hepatology 34: 255-61
72. Saliba F, Ichai P, Levesque E, Samuel D, (2013) Cirrhotic patients in the ICU: prognostic markers and outcome. Curr Opin Crit Care 19: 154-160

73. European Association for the Study of the Liver. Electronic address: easloffice@easloffice.eu., (2016) EASL Clinical Practice Guidelines: Liver transplantation. J Hepatol 64: 433-485

74. Artru F, Louvet A, Ruiz I, Levesque E, Labreuche J, Ursic-Bedoya J, Lassailly G, Dharancy S, Boleslawski E, Lebuffe G, Kipnis E, Ichai P, Coilly A, De Martin E, Antonini TM, Vibert E, Jaber S, Herrerro A, Samuel D, Duhamel A, Pageaux GP, Mathurin P, Saliba F, (2017) Liver transplantation in the most severely ill cirrhotic patients: a multicenter study in acute-on-chronic liver failure grade 3. J Hepatol 67: 708-715

75. Huebener P, Sterneck MR, Bangert K, Drolz A, Lohse AW, Kluge S, Fischer L, Fuhrmann V, (2018) Stabilisation of acuteon-chronic liver failure patients before liver transplantation predicts post-transplant survival. Aliment Pharmacol Ther 47: $1502-1510$

76. Thuluvath PJ, Thuluvath AJ, Hanish S, Savva Y, (2018) Liver transplantation in patients with multiple organ failures: Feasibility and outcomes. J Hepatol 69: 1047-1056 (doi: 10.1016/j. jhep.2018.07.007. Epub 2018 Jul 31) 